\title{
Mapping Properties of Some Classes of Analytic Functions under Certain Integral Operators
}

\author{
Serap Bulut \\ Civil Aviation College, Kocaeli University, Arslanbey Campus, 41285 Kocaeli, Turkey \\ Correspondence should be addressed to Serap Bulut; bulutserap@yahoo.com
}

Received 15 November 2012; Accepted 3 January 2013

Academic Editor: Abdellatif Agouzal

Copyright (C) 2013 Serap Bulut. This is an open access article distributed under the Creative Commons Attribution License, which permits unrestricted use, distribution, and reproduction in any medium, provided the original work is properly cited.

We consider certain subclasses of analytic functions with bounded radius and bounded boundary rotation and study the mapping properties of these classes under certain integral operators.

\section{Introduction}

Let $\mathscr{A}$ be the class of all functions of the following form:

$$
f(z)=z+\sum_{k=2}^{\infty} a_{k} z^{k}
$$

which are analytic in the open unit disc

$$
\mathbb{U}=\{z \in \mathbb{C}:|z|<1\} .
$$

A function $f \in \mathscr{A}$ is said to be spiral-like if there exists a real number $\lambda(|\lambda|<\pi / 2)$ such that

$$
\operatorname{Re}\left\{e^{i \lambda} \frac{z f^{\prime}(z)}{f(z)}\right\}>0 \quad(z \in \mathbb{U}) .
$$

The class of all spiral-like functions was introduced by Spacek [1] in 1933 and we denote it by $\mathcal{S}_{\lambda}^{*}$. Later in 1969, Robertson [2] considered the class $\mathscr{C}_{\lambda}$ of analytic functions in $\mathbb{U}$ for which $z f^{\prime}(z) \in \mathcal{S}_{\lambda}^{*}$.

Let $\mathscr{P}_{k}^{\lambda}(\rho)$ be the class of functions $p(z)$ analytic in $\mathbb{U}$ with $p(0)=1$ and

$$
\int_{0}^{2 \pi}\left|\frac{\operatorname{Re} e^{i \lambda} p(z)-\rho \cos \lambda}{1-\rho}\right| d \theta \leq k \pi \cos \lambda, \quad z=r e^{i \theta},
$$

where $k \geq 2,0 \leq \rho<1, \lambda$ is real with $|\lambda|<\pi / 2$.

For $\lambda=0$, this class was introduced in [3] and for $\rho=0$, see [4]. For $k=2, \lambda=0$ and $\rho=0$, the class $\mathscr{P}_{k}^{\lambda}(\rho)$ reduces to the class $\mathscr{P}$ of functions $p(z)$ analytic in $\mathbb{U}$ with $p(0)=1$ and whose real part is positive.

The following definition of fractional derivative by Owa [5] (also by Srivastava and Owa [6]) will be required in our investigation.

The fractional derivative of order $\gamma$ is defined, for a function $f$, by

$$
D_{z}^{\gamma} f(z)=\frac{1}{\Gamma(1-\gamma)} \frac{d}{d z} \int_{0}^{z} \frac{f(\xi)}{(z-\xi)^{\gamma}} d \xi \quad(0 \leq \gamma<1),
$$

where the function $f$ is analytic in a simply connected region of the complex $z$-plane containing the origin, and the multiplicity of $(z-\xi)^{-\gamma}$ is removed by requiring $\log (z-\xi)$ to be real when $z-\xi>0$.

It readily follows from (5) that

$$
D_{z}^{\gamma} z^{k}=\frac{\Gamma(k+1)}{\Gamma(k+1-\gamma)} z^{k-\gamma} \quad(0 \leq \gamma<1, k \in \mathbb{N}=\{1,2, \ldots\}) .
$$

Using $D_{z}^{\gamma} f$, Owa and Srivastava [7] introduced the operator $\Omega^{\gamma}: \mathscr{A} \rightarrow \mathscr{A}$, which is known as an extension of fractional derivative and fractional integral, as follows:

$$
\begin{aligned}
\Omega^{\gamma} f(z) & =\Gamma(2-\gamma) z^{\gamma} D_{z}^{\gamma} f(z) \\
& =z+\sum_{k=2}^{\infty} \frac{\Gamma(k+1) \Gamma(2-\gamma)}{\Gamma(k+1-\gamma)} a_{k} z^{k} .
\end{aligned}
$$


Note that

$$
\Omega^{0} f(z)=f(z) .
$$

In [8], Al-Oboudi and Al-Amoudi defined the linear multiplier fractional differential operator (namely, generalized Al-Oboudi differential operator) $D_{\delta}^{n, \gamma}$ as follows:

$$
\begin{gathered}
D^{0} f(z)=f(z), \\
D_{\delta}^{1, \gamma} f(z)=(1-\delta) \Omega^{\gamma} f(z)+\delta z\left(\Omega^{\gamma} f(z)\right)^{\prime} \\
=D_{\delta}^{\gamma}(f(z)), \quad \delta \geq 0,0 \leq \gamma<1, \\
D_{\delta}^{2, \gamma} f(z)=D_{\delta}^{\gamma}\left(D_{\delta}^{1, \gamma} f(z)\right), \\
\vdots \\
D_{\delta}^{n, \gamma} f(z)=D_{\delta}^{\gamma}\left(D_{\delta}^{n-1, \gamma} f(z)\right), \quad n \in \mathbb{N} .
\end{gathered}
$$

If $f$ is given by (1), then by (7) and (9), we see that

$$
D_{\delta}^{n, \gamma} f(z)=z+\sum_{k=2}^{\infty} \Psi_{k, n}(\gamma, \delta) a_{k} z^{k}, \quad n \in \mathbb{N}_{0}=\mathbb{N} \cup\{0\}
$$

where

$$
\Psi_{k, n}(\gamma, \delta)=\left[\frac{\Gamma(k+1) \Gamma(2-\gamma)}{\Gamma(k+1-\gamma)}(1+(k-1) \delta)\right]^{n} .
$$

Remark 1. (i) When $\gamma=0$, we get Al-Oboudi differential operator [9].

(ii) When $\gamma=0$ and $\delta=1$, we get Sălăgean differential operator [10].

(iii) When $n=1$ and $\delta=0$, we get Owa-Srivastava fractional differential operator [7].

Definition 2. A function $f \in \mathscr{A}$ is said to belong to the class $\mathscr{R}_{k}^{\lambda}(\rho, b ; n, \gamma, \delta)$ if and only if

$$
1+\frac{1}{b}\left(\frac{z\left(D_{\delta}^{n, \gamma} f(z)\right)^{\prime}}{D_{\delta}^{n, \gamma} f(z)}-1\right) \in \mathscr{P}_{k}^{\lambda}(\rho),
$$

where $k \geq 2,0 \leq \rho<1, \lambda$ is real with $|\lambda|<\pi / 2, b \in \mathbb{C}-\{0\}$ and $D$ is the generalized Al-Oboudi differential operator.

Definition 3. A function $f \in \mathscr{A}$ is said to belong to the class $\mathscr{V}_{k}^{\lambda}(\rho, b ; n, \gamma, \delta)$ if and only if

$$
1+\frac{1}{b} \frac{z\left(D_{\delta}^{n, \gamma} f(z)\right)^{\prime \prime}}{\left(D_{\delta}^{n, \gamma} f(z)\right)^{\prime}} \in \mathscr{P}_{k}^{\lambda}(\rho)
$$

where $k \geq 2,0 \leq \rho<1, \lambda$ is real with $|\lambda|<\pi / 2, b \in \mathbb{C}-\{0\}$ and $D$ is the generalized Al-Oboudi differential operator.

Remark 4. (i) Letting $\gamma=0$ and $b=1$ in Definition 2, we have the class $\mathscr{V}_{k}^{\lambda}(\rho, \delta, n)$ introduced by Dileep and Latha [11]. (ii) For $n=0$ and $b=1$, we obtain the classes $\mathscr{R}_{k}^{\lambda}(\rho)$ and $\mathscr{V}_{k}^{\lambda}(\rho)$, respectively, introduced and studied by Noor et al. [12] and Moulis [13].

(iii) For $\lambda=0$ and $n=0$, we have the classes $\mathscr{R}_{k}(\rho, b)$ and $\mathscr{V}_{k}(\rho, b)$, respectively, introduced and studied by Noor et al. [14].

(iv) For $k=2, \lambda=0$ and $n=0$, we have the classes $\delta_{\rho}^{*}(b)$ and $\mathscr{C}_{\rho}(b)$, respectively, introduced by Frasin [15].

Definition 5. Let $n \in \mathbb{N}_{0}, l=\left(l_{1}, \ldots, l_{m}\right) \in \mathbb{N}_{0}^{m}$, and $k_{j}>$ $0(1 \leq j \leq m)$. One defines the integral operator $I_{n, m, l, k}$ : $\mathscr{A}^{m} \rightarrow \mathscr{A}$ as

$$
\begin{aligned}
& I_{n, m, l, k}\left(f_{1}, \ldots, f_{m}\right)=F, \\
D_{\delta}^{n, \gamma} F(z)=\int_{0}^{z}\left(\frac{D_{\delta}^{l_{1}, \gamma} f_{1}(t)}{t}\right)^{k_{1}} & \\
& \ldots\left(\frac{D_{\delta}^{l_{m}, \gamma} f_{m}(t)}{t}\right)^{k_{m}} d t \quad(z \in \mathbb{U}),
\end{aligned}
$$

where $f_{1}, \ldots, f_{m} \in \mathscr{A}$ and $D$ is the generalized Al-Oboudi differential operator.

Remark 6. The integral operator $D_{\delta}^{n, \gamma} F$ generalizes many operators which were introduced and studied recently.

(i) For $\gamma=0$, we have the integral operator

$$
D_{\delta}^{n} F(z)=\int_{0}^{z}\left(\frac{D_{\delta}^{l_{1}} f_{1}(t)}{t}\right)^{k_{1}} \ldots\left(\frac{D_{\delta}^{l_{m}} f_{m}(t)}{t}\right)^{k_{m}} d t
$$

introduced by Bulut [16]. Here D is the Al-Oboudi differential operator.

(ii) For $n=0, \gamma=0$ and $l_{1}=\cdots=l_{m}=l \in \mathbb{N}_{0}$, we have the integral operator

$$
F(z)=\int_{0}^{z}\left(\frac{D_{\delta}^{l} f_{1}(t)}{t}\right)^{k_{1}} \cdots\left(\frac{D_{\delta}^{l} f_{m}(t)}{t}\right)^{k_{m}} d t
$$

introduced by Bulut [17]. Here D is the Al-Oboudi differential operator.

(iii) For $\gamma=0$ and $\lambda=1$, we have the integral operator

$$
D^{n} F(z)=\int_{0}^{z}\left(\frac{D^{l_{1}} f_{1}(t)}{t}\right)^{k_{1}} \ldots\left(\frac{D^{l_{m}} f_{m}(t)}{t}\right)^{k_{m}} d t
$$

introduced by Breaz et al. [18]. Here $D$ is the Sălăgean differential operator.

(iv) For $n=0$ and $D_{\delta}^{0, \gamma} f_{j}=D_{0}^{1,0} f_{j}=f_{j} \in \mathscr{A}(1 \leq j \leq$ $m)$, we have the integral operator

$$
F_{m}(z)=\int_{0}^{z}\left(\frac{f_{1}(t)}{t}\right)^{k_{1}} \cdots\left(\frac{f_{m}(t)}{t}\right)^{k_{m}} d t
$$

introduced by D. Breaz and N. Breaz [19]. 
(v) For $n=0, m=1, k_{1}=k \in[0,1], k_{2}=\cdots=k_{m}=$ 0 and $D_{\delta}^{0, \gamma} f_{1}=D_{0}^{1,0} f_{1}=f \in \mathcal{S}^{*}$ (consists of functions that are analytic, univalent and starlike), we have the integral operator

$$
F_{k}(z)=\int_{0}^{z}\left(\frac{f(t)}{t}\right)^{k} d t
$$

studied by Miller et al. [20].

(vi) For $n=0, m=1, k_{1}=1, k_{2}=\cdots=k_{m}=0$ and $D_{\delta}^{0, \gamma} f_{1}=D_{0}^{1,0} f_{1}=f \in \mathscr{A}$, we have the integral operator of Alexander

$$
F(z)=\int_{0}^{z} \frac{f(t)}{t} d t
$$

introduced by Alexander [21].

Definition 7. Let $n \in \mathbb{N}_{0}, l=\left(l_{1}, \ldots, l_{m}\right) \in \mathbb{N}_{0}^{m}$, and $k_{j}>$ $0(1 \leq j \leq m)$. One defines the integral operator $J_{n, m, l, k}$ : $\mathscr{A}^{m} \rightarrow \mathscr{A}$ as

$$
\begin{gathered}
J_{n, m, l, k}\left(f_{1}, \ldots, f_{m}\right)=G \\
D_{\delta}^{n, \gamma} G(z)=\int_{0}^{z}\left[\left(D_{\delta}^{l_{1}, \gamma} f_{1}(t)\right)^{\prime}\right]^{k_{1}} \\
\ldots\left[\left(D_{\delta}^{l_{m}, \gamma} f_{1}(t)\right)^{\prime}\right]^{k_{m}} d t \quad(z \in \mathbb{U}),
\end{gathered}
$$

where $f_{1}, \ldots, f_{m} \in \mathscr{A}$ and $D$ is the generalized Al-Oboudi differential operator.

Remark 8. The integral operator $D_{\delta}^{n, \gamma} G$ generalizes many operators which were introduced and studied recently.

(i) For $n=0$ and $D_{\delta}^{0, \gamma} f_{j}=D_{0}^{1,0} f_{j}=f_{j} \in \mathscr{A}(1 \leq j \leq$ $m$ ), we have the integral operator

$$
G(z)=\int_{0}^{z}\left(f_{1}^{\prime}(t)\right)^{k_{1}} \cdots\left(f_{m}^{\prime}(t)\right)^{k_{m}} d t
$$

introduced by Breaz et al. [22].

(ii) For $n=0, m=1, k_{1}=k \in \mathbb{C}, k_{2}=\cdots=k_{m}=0$ and $D_{\delta}^{0, \gamma} f_{1}=D_{0}^{1,0} f_{1}=f \in \mathscr{A}$, we have the integral operator

$$
G_{k}(z)=\int_{0}^{z}\left(f^{\prime}(t)\right)^{k} d t
$$

introduced by Pfaltzgraff [23] (see also Pascu and Pescar [24]).

In this paper, we investigate some propeties of the above integral operators $D_{\delta}^{n, \gamma} F$ and $D_{\delta}^{n, \gamma} G$ for the classes

$$
\mathscr{R}_{k}^{\lambda}(\rho, b ; n, \gamma, \delta), \quad \mathscr{V}_{k}^{\lambda}(\rho, b ; n, \gamma, \delta) .
$$

\section{Main Results}

Theorem 9. Let $f_{j} \in \mathscr{R}_{k}^{\lambda}\left(\rho_{j}, b ; n, \gamma, \delta\right)$ for $1 \leq j \leq m$ with $0 \leq \rho_{j}<1, b \in \mathbb{C}-\{0\}$. Also let $\lambda$ be real with $|\lambda|<\pi / 2, k_{j}>$ $0(1 \leq j \leq m)$. If

$$
0 \leq 1+\sum_{j=1}^{m} k_{j}\left(\rho_{j}-1\right)<1,
$$

then the integral operator $F$ defined by (15) is in the class $\mathscr{V}_{k}^{\lambda}(\eta, b ; n, \gamma, \delta)$ with

$$
\eta=1+\sum_{j=1}^{m} k_{j}\left(\rho_{j}-1\right)
$$

Proof. Since $f_{j} \in \mathscr{A}(1 \leq j \leq m)$, by (10), we have

$$
\begin{aligned}
& \frac{D_{\delta}^{l_{j}, \gamma} f_{j}(z)}{z}=1+\sum_{k=2}^{\infty} \Psi_{k, l_{j}}(\gamma, \delta) a_{k, j} z^{k-1}, \\
& \frac{D_{\delta}^{l_{j}, \gamma} f_{j}(z)}{z} \neq 0
\end{aligned}
$$

for all $z \in \mathbb{U}$. By (15), we get

$$
\left(D_{\delta}^{n, \gamma} F(z)\right)^{\prime}=\left(\frac{D_{\delta}^{l_{1}, \gamma} f_{1}(z)}{z}\right)^{k_{1}} \ldots\left(\frac{D_{\delta}^{l_{m}, \gamma} f_{m}(z)}{z}\right)^{k_{m}} .
$$

This equality implies that

$$
\ln \left(D_{\delta}^{n, \gamma} F(z)\right)^{\prime}=k_{1} \ln \frac{D_{\delta}^{l_{1}, \gamma} f_{1}(z)}{z}+\cdots+k_{m} \ln \frac{D_{\delta}^{l_{m}, \gamma} f_{m}(z)}{z}
$$

or equivalently

$$
\begin{aligned}
\ln \left(D_{\delta}^{n, \gamma} F(z)\right)^{\prime}= & k_{1}\left[\ln D_{\delta}^{l_{1}, \gamma} f_{1}(z)-\ln z\right] \\
& +\cdots+k_{m}\left[\ln D_{\delta}^{l_{m}, \gamma} f_{m}(z)-\ln z\right] .
\end{aligned}
$$

By differentiating the above equality, we get

$$
\frac{\left(D_{\delta}^{n, \gamma} F(z)\right)^{\prime \prime}}{\left(D_{\delta}^{n, \gamma} F(z)\right)^{\prime}}=\sum_{j=1}^{m} k_{j}\left[\frac{\left(D_{\delta}^{l_{j}, \gamma} f_{j}(z)\right)^{\prime}}{D_{\delta}^{l_{j}, \gamma} f_{j}(z)}-\frac{1}{z}\right] .
$$

Hence, we obtain from this equality that

$$
\frac{z\left(D_{\delta}^{n, \gamma} F(z)\right)^{\prime \prime}}{\left(D_{\delta}^{n, \gamma} F(z)\right)^{\prime}}=\sum_{j=1}^{m} k_{j}\left(\frac{z\left(D_{\delta}^{l_{j}, \gamma} f_{j}(z)\right)^{\prime}}{D_{\delta}^{l_{j}, \gamma} f_{j}(z)}-1\right) .
$$


Then by multiplying the above relation with $1 / b$, we have

$$
\begin{aligned}
& \frac{1}{b} \frac{z\left(D_{\delta}^{n, \gamma} F(z)\right)^{\prime \prime}}{\left(D_{\delta}^{n, \gamma} F(z)\right)^{\prime}} \\
& \quad=\sum_{j=1}^{m} k_{j} \frac{1}{b}\left(\frac{z\left(D_{\delta}^{l_{j}, \gamma} f_{j}(z)\right)^{\prime}}{D_{\delta}^{l_{j}, \gamma} f_{j}(z)}-1\right) \\
& \quad=\sum_{j=1}^{m} k_{j}\left[1+\frac{1}{b}\left(\frac{z\left(D_{\delta}^{l_{j}, \gamma} f_{j}(z)\right)^{\prime}}{D_{\delta}^{l_{j}, \gamma} f_{j}(z)}-1\right)\right]-\sum_{j=1}^{m} k_{j}
\end{aligned}
$$

or equivalently

$$
\begin{aligned}
e^{i \lambda}\left(1+\frac{1}{b} \frac{z\left(D_{\delta}^{n, \gamma} F(z)\right)^{\prime \prime}}{\left(D_{\delta}^{n, \gamma} F(z)\right)^{\prime}}\right) \\
=\left(1-\sum_{j=1}^{m} k_{j}\right) e^{i \lambda} \\
+\sum_{j=1}^{m} k_{j} e^{i \lambda}\left[1+\frac{1}{b}\left(\frac{z\left(D_{\delta}^{l_{j}, \gamma} f_{j}(z)\right)^{\prime}}{D_{\delta}^{l_{j}, \gamma} f_{j}(z)}-1\right)\right] .
\end{aligned}
$$

Subtracting and adding $\cos \lambda \sum_{j=1}^{m} k_{j} \rho_{j}$ on the left hand side and then taking real part, we have

$$
\begin{gathered}
\operatorname{Re}\left\{e^{i \lambda}\left(1+\frac{1}{b} \frac{z\left(D_{\delta}^{n, \gamma} F(z)\right)^{\prime \prime}}{\left(D_{\delta}^{n, \gamma} F(z)\right)^{\prime}}\right)-\eta \cos \lambda\right\} \\
=\sum_{j=1}^{m} k_{j} \operatorname{Re}\left\{e^{i \lambda}\left[1+\frac{1}{b}\left(\frac{z\left(D_{\delta}^{l_{j}, \gamma} f_{j}(z)\right)^{\prime}}{D_{\delta}^{l_{j}, \gamma} f_{j}(z)}-1\right)\right]\right. \\
\left.-\rho_{j} \cos \lambda\right\},
\end{gathered}
$$

where $\eta$ is given by (28). Integrating (37) and then using (28), we have

$$
\begin{gathered}
\int_{0}^{2 \pi}\left|\operatorname{Re}\left\{e^{i \lambda}\left(1+\frac{1}{b} \frac{z\left(D_{\delta}^{n, \gamma} F(z)\right)^{\prime \prime}}{\left(D_{\delta}^{n, \gamma} F(z)\right)^{\prime}}\right)-\eta \cos \lambda\right\}\right| d \theta \\
\leq \sum_{j=1}^{m} k_{j} \int_{0}^{2 \pi} \mid \operatorname{Re}\left\{e^{i \lambda}\left[1+\frac{1}{b}\left(\frac{z\left(D_{\delta}^{l_{j}, \gamma} f_{j}(z)\right)^{\prime}}{D_{\delta}^{l_{j}, \gamma} f_{j}(z)}-1\right)\right]\right. \\
\left.-\rho_{j} \cos \lambda\right\} \mid d \theta .
\end{gathered}
$$

Since $f_{j} \in \mathscr{R}_{k}^{\lambda}\left(\rho_{j}, b ; n, \gamma, \delta\right)(1 \leq j \leq m)$, we get

$$
\begin{array}{r}
\int_{0}^{2 \pi} \mid \operatorname{Re}\left\{e^{i \lambda}\left[1+\frac{1}{b}\left(\frac{z\left(D_{\delta}^{l_{j}, \gamma} f_{j}(z)\right)^{\prime}}{D_{\delta}^{l_{j}, \gamma} f_{j}(z)}-1\right)\right]\right. \\
\left.-\rho_{j} \cos \lambda\right\} \mid d \theta \leq\left(1-\rho_{j}\right) k \pi \cos \lambda
\end{array}
$$

for $1 \leq j \leq m$. Using (39) in (38), we obtain

$$
\begin{aligned}
& \int_{0}^{2 \pi}\left|\operatorname{Re}\left\{e^{i \lambda}\left(1+\frac{1}{b} \frac{z\left(D_{\delta}^{n, \gamma} F(z)\right)^{\prime \prime}}{\left(D_{\delta}^{n, \gamma} F(z)\right)^{\prime}}\right)-\eta \cos \lambda\right\}\right| d \theta \\
& \leq k \pi \cos \lambda \sum_{j=1}^{m} k_{j}\left(1-\rho_{j}\right) .
\end{aligned}
$$

Hence, we obtain $F \in \mathscr{V}_{k}^{\lambda}(\eta, b ; n, \gamma, \delta)$ with $\eta$ is given by (28).

By setting $n=0, \gamma=0, b=1, l_{1}=\cdots=l_{m}=l$ in Theorem 9 , we obtain the following.

Corollary 10 (see [11, Theorem 1]). Let $f_{j} \in \mathscr{V}_{k}^{\lambda}\left(\rho_{j}, \delta, n\right)$ for $1 \leq j \leq m$ with $0 \leq \rho_{j}<1$. Also let $\lambda$ be real with $|\lambda|<$ $\pi / 2, k_{j}>0(1 \leq j \leq m)$. If

$$
0 \leq 1+\sum_{j=1}^{m} k_{j}\left(\rho_{j}-1\right)<1,
$$

then the integral operator $F(z)$ defined by (17) is in the class $\mathscr{V}_{k}^{\lambda}(\eta)$ with

$$
\eta=1+\sum_{j=1}^{m} k_{j}\left(\rho_{j}-1\right) .
$$


Remark 11. Letting $\rho_{1}=\rho_{2}=\cdots=\rho_{m}=\rho$ in Corollary 10, then we have [12, Theorem 3.1].

By setting $n=0, \lambda=0$ and $D_{\delta}^{0, \gamma}=D_{0}^{1,0}$ in Theorem 9, we obtain the following.

Corollary 12. Let $f_{j} \in \mathscr{R}_{k}\left(\rho_{j}, b\right)$ for $1 \leq j \leq m$ with $0 \leq$ $\rho_{j}<1, b \in \mathbb{C}-\{0\}$. Also let $k_{j}>0(1 \leq j \leq m)$. If

$$
0 \leq 1+\sum_{j=1}^{m} k_{j}\left(\rho_{j}-1\right)<1,
$$

then the integral operator $F_{m}(z)$ defined by (19) is in the class $\mathscr{V}_{k}(\eta, b)$ with

$$
\eta=1+\sum_{j=1}^{m} k_{j}\left(\rho_{j}-1\right)
$$

Remark 13. In Corollary 12, letting

(i) $\rho_{1}=\rho_{2}=\cdots=\rho_{m}=\rho$, we have [14, Theorem 2.1],

(ii) $k=2$, we have [25, Theorem 1].

Theorem 14. Let $f_{j} \in \mathscr{V}_{k}^{\lambda}\left(\rho_{j}, b ; n, \gamma, \delta\right)$ for $1 \leq j \leq m$ with $0 \leq \rho_{j}<1, b \in \mathbb{C}-\{0\}$. Also let $\lambda$ be real with $|\lambda|<\pi / 2, k_{j}>$ $0(1 \leq j \leq m)$. If

$$
0 \leq 1+\sum_{j=1}^{m} k_{j}\left(\rho_{j}-1\right)<1,
$$

then the integral operator $G$ defined by (23) is in the class $\mathscr{V}_{k}^{\lambda}(\mu, b ; n, \gamma, \delta)$ with

$$
\mu=1+\sum_{j=1}^{m} k_{j}\left(\rho_{j}-1\right)
$$

Proof. By (23), we get

$$
\begin{aligned}
& \left(D_{\delta}^{n, \gamma} G(z)\right)^{\prime} \\
& \quad=\left[\left(D_{\delta}^{l_{1}, \gamma} f_{1}(z)\right)^{\prime}\right]^{k_{1}} \ldots\left[\left(D_{\delta}^{l_{m}, \gamma} f_{1}(z)\right)^{\prime}\right]^{k_{m}} .
\end{aligned}
$$

This equality implies that

$$
\begin{aligned}
& \left(D_{\delta}^{n, \gamma} G(z)\right)^{\prime \prime} \\
& =\sum_{j=1}^{m} k_{j}\left[\left(D_{\delta}^{l_{j}, \gamma} f_{j}(z)\right)^{\prime}\right]^{k_{j}} \frac{\left(D_{\delta}^{l_{j}, \gamma} f_{j}(z)\right)^{\prime \prime}}{\left(D_{\delta}^{l_{j}, \gamma} f_{j}(z)\right)^{\prime}} \\
& \quad \times \prod_{\substack{r=1 \\
(r \neq j)}}^{m}\left[\left(D_{\delta}^{l_{r}, \gamma} f_{r}(z)\right)^{\prime}\right]^{k_{r}} .
\end{aligned}
$$

Thus by using (47) and (48), we obtain

$$
\frac{z\left(D_{\delta}^{n, \gamma} G(z)\right)^{\prime \prime}}{\left(D_{\delta}^{n, \gamma} G(z)\right)^{\prime}}=\sum_{j=1}^{m} k_{j} \frac{z\left(D_{\delta}^{l_{j}, \gamma} f_{j}(z)\right)^{\prime \prime}}{\left(D_{\delta}^{l_{j}, \gamma} f_{j}(z)\right)^{\prime}} .
$$

Then by multiplying the above relation with $1 / b$, we have

$$
\begin{aligned}
& \frac{1}{b} \frac{z\left(D_{\delta}^{n, \gamma} G(z)\right)^{\prime \prime}}{\left(D_{\delta}^{n, \gamma} G(z)\right)^{\prime}} \\
& \quad=\sum_{j=1}^{m} k_{j} \frac{1}{b} \frac{z\left(D_{\delta}^{l_{j}, \gamma} f_{j}(z)\right)^{\prime \prime}}{\left(D_{\delta}^{l_{j}, \gamma} f_{j}(z)\right)^{\prime}} \\
& =\sum_{j=1}^{m} k_{j}\left(1+\frac{1}{b} \frac{z\left(D_{\delta}^{l_{j}, \gamma} f_{j}(z)\right)^{\prime \prime}}{\left(D_{\delta}^{l_{j}, \gamma} f_{j}(z)\right)^{\prime}}\right)-\sum_{j=1}^{n} k_{j}
\end{aligned}
$$

or equivalently

$$
\begin{aligned}
e^{i \lambda}\left(1+\frac{1}{b} \frac{z\left(D_{\delta}^{n, \gamma} G(z)\right)^{\prime \prime}}{\left(D_{\delta}^{n, \gamma} G(z)\right)^{\prime}}\right) \\
=\left(1-\sum_{j=1}^{m} k_{j}\right) e^{i \lambda} \\
+\sum_{j=1}^{m} k_{j} e^{i \lambda}\left(1+\frac{1}{b} \frac{z\left(D_{\delta}^{l_{j}, \gamma} f_{j}(z)\right)^{\prime \prime}}{\left(D_{\delta}^{l_{j}, \gamma} f_{j}(z)\right)^{\prime}}\right) .
\end{aligned}
$$

Subtracting and adding $\cos \lambda \sum_{j=1}^{m} k_{j} \rho_{j}$ on the left hand side and then taking real part, we have

$$
\begin{aligned}
& \operatorname{Re}\left\{e^{i \lambda}\left(1+\frac{1}{b} \frac{z\left(D_{\delta}^{n, \gamma} G(z)\right)^{\prime \prime}}{\left(D_{\delta}^{n, \gamma} G(z)\right)^{\prime}}\right)-\mu \cos \lambda\right\} \\
& =\sum_{j=1}^{m} k_{j} \operatorname{Re}\left\{e^{i \lambda}\left(1+\frac{1}{b} \frac{z\left(D_{\delta}^{l_{j}, \gamma} f_{j}(z)\right)^{\prime \prime}}{\left(D_{\delta}^{l_{j}, \gamma} f_{j}(z)\right)^{\prime}}\right)-\rho_{j} \cos \lambda\right\},
\end{aligned}
$$


where $\mu$ is given by (46). Integrating (52) and then using (46), we have

$$
\begin{gathered}
\int_{0}^{2 \pi}\left|\operatorname{Re}\left\{e^{i \lambda}\left(1+\frac{1}{b} \frac{z\left(D_{\delta}^{n, \gamma} G(z)\right)^{\prime \prime}}{\left(D_{\delta}^{n, \gamma} G(z)\right)^{\prime}}\right)-\mu \cos \lambda\right\}\right| d \theta \\
\leq \sum_{j=1}^{m} k_{j} \int_{0}^{2 \pi} \mid \operatorname{Re}\left\{e^{i \lambda}\left(1+\frac{1}{b} \frac{z\left(D_{\delta}^{l_{j}, \gamma} f_{j}(z)\right)^{\prime \prime}}{\left(D_{\delta}^{l_{j}, \gamma} f_{j}(z)\right)^{\prime}}\right)\right. \\
\left.-\rho_{j} \cos \lambda\right\} \mid d \theta .
\end{gathered}
$$

Since $f_{j} \in \mathscr{V}_{k}^{\lambda}\left(\rho_{j}, b ; n, \gamma, \delta\right)(1 \leq j \leq m)$, we get

$$
\begin{aligned}
& \int_{0}^{2 \pi}\left|\operatorname{Re}\left\{e^{i \lambda}\left(1+\frac{1}{b} \frac{z\left(D_{\delta}^{l_{j}, \gamma} f_{j}(z)\right)^{\prime \prime}}{\left(D_{\delta}^{l_{j}, \gamma} f_{j}(z)\right)^{\prime}}\right)-\rho_{j} \cos \lambda\right\}\right| d \theta \\
& \quad \leq\left(1-\rho_{j}\right) k \pi \cos \lambda
\end{aligned}
$$

for $1 \leq j \leq m$. Using (54) in (53), we obtain

$$
\begin{aligned}
& \int_{0}^{2 \pi}\left|\operatorname{Re}\left\{e^{i \lambda}\left(1+\frac{1}{b} \frac{z\left(D_{\delta}^{n, \gamma} G(z)\right)^{\prime \prime}}{\left(D_{\delta}^{n, \gamma} G(z)\right)^{\prime}}\right)-\mu \cos \lambda\right\}\right| d \theta \\
& \leq k \pi \cos \lambda \sum_{j=1}^{m} k_{j}\left(1-\rho_{j}\right) .
\end{aligned}
$$

Hence, we obtain $G \in \mathscr{V}_{k}^{\lambda}(\mu, b ; n, \gamma, \delta)$ with $\mu$ given by (46).

By setting $n=0, \lambda=0$ and $D_{\delta}^{0, \gamma}=D_{0}^{1,0}$ in Theorem 14 , we obtain the following.

Corollary 15. Let $f_{j} \in \mathscr{V}_{k}\left(\rho_{j}, b\right)$ for $1 \leq j \leq m$ with $0 \leq$ $\rho_{j}<1, b \in \mathbb{C}-\{0\}$. Also let $k_{j}>0(1 \leq j \leq m)$. If

$$
0 \leq 1+\sum_{j=1}^{m} k_{j}\left(\rho_{j}-1\right)<1,
$$

then the integral operator $G(z)$ defined by (24) is in the class $\mathscr{V}_{k}(\mu, b)$ with

$$
\mu=1+\sum_{j=1}^{m} k_{j}\left(\rho_{j}-1\right) .
$$

Remark 16. In Corollary 15, letting

(i) $\rho_{1}=\rho_{2}=\cdots=\rho_{m}=\rho$, we have [14, Theorem 2.5],

(ii) $k=2$, we have [25, Theorem 3].

\section{References}

[1] L. Spacek, "Prispěvek k teorii funkei prostych," Čapopis pro pěstováni matematiky a fysiky, vol. 62, pp. 12-19, 1933.

[2] M. S. Robertson, "Univalent functions $f(z)$ for which $z f(z)$ is spirallike," The Michigan Mathematical Journal, vol. 16, pp. 97-101, 1969.

[3] K. S. Padmanabhan and R. Parvatham, "Properties of a class of functions with bounded boundary rotation," Annales Polonici Mathematici, vol. 31, no. 3, pp. 311-323, 1975.

[4] B. Pinchuk, "Functions of bounded boundary rotation," Israel Journal of Mathematics, vol. 10, pp. 7-16, 1971.

[5] S. Owa, "On the distortion theorems. I," Kyungpook Mathematical Journal, vol. 18, no. 1, pp. 53-59, 1978.

[6] H. M. Srivastava and S. Owa, Eds., Univalent Functions, Fractional Calculus, and Their Applications, Ellis Horwood Series: Mathematics and its Applications, Ellis Horwood, Chichester, UK, John Wiley \& Sons, New York, NY, USA, 1989.

[7] S. Owa and H. M. Srivastava, "Univalent and starlike generalized hypergeometric functions," Canadian Journal of Mathematics, vol. 39, no. 5, pp. 1057-1077, 1987.

[8] F. M. Al-Oboudi and K. A. Al-Amoudi, "On classes of analytic functions related to conic domains," Journal of Mathematical Analysis and Applications, vol. 339, no. 1, pp. 655-667, 2008.

[9] F. M. Al-Oboudi, "On univalent functions defined by a generalized Sălăgean operator," International Journal of Mathematics and Mathematical Sciences, no. 25-28, pp. 1429-1436, 2004.

[10] G. Ş. Sălăgean, "Subclasses of univalent functions," in Complex Analysis-Fifth Romanian-Finnish seminar. Part 1, vol. 1013 of Lecture Notes in Mathematics, pp. 362-372, Springer, Berlin, Germany, 1983.

[11] L. Dileep and S. Latha, "A generalized integral operator associated with functions of bounded boundary rotation," General Mathematics, vol. 19, no. 3, pp. 25-30, 2011.

[12] K. I. Noor, M. Arif, and A. Muhammad, "Mapping properties of some classes of analytic functions under an integral operator," Journal of Mathematical Inequalities, vol. 4, no. 4, pp. 593-600, 2010.

[13] E. J. Moulis Jr., "Generalizations of the Robertson functions," Pacific Journal of Mathematics, vol. 81, no. 1, pp. 167-174, 1979.

[14] K. I. Noor, M. Arif, and W. U. Haq, "Some properties of certain integral operators," Acta Universitatis Apulensis. Mathematics. Informatics, no. 21, pp. 89-95, 2010.

[15] B. A. Frasin, "Family of analytic functions of complex order," Acta Mathematica Academiae Paedagogiace Nyíregyháziensis (N. S.), vol. 22, no. 2, pp. 179-191, 2006.

[16] S. Bulut, "A new general integral operator defined by Al-Oboudi differential operator," Journal of Inequalities and Applications, vol. 2009, Article ID 158408, 13 pages, 2009.

[17] S. Bulut, "Some properties for an integral operator defined by Al-Oboudi differential operator," Journal of Inequalities in Pure and Applied Mathematics, vol. 9, no. 4, article 115, 5 pages, 2008.

[18] D. Breaz, H. Ö. Güney, and G. Ş. Sălăgean, "A new general integral operator," Tamsui Oxford Journal of Mathematical Sciences, vol. 25, no. 4, pp. 407-414, 2009.

[19] D. Breaz and N. Breaz, "Two integral operators," Studia Universitatis Babes-Bolyai Mathematica, vol. 47, no. 3, pp. 13-19, 2002.

[20] S. S. Miller, P. T. Mocanu, and M. O. Reade, "Starlike integral operators," Pacific Journal of Mathematics, vol. 79, no. 1, pp. $157-168,1978$. 
[21] I. W. Alexander, "Functions which map the interior of the unit circle upon simple regions," Annals of Mathematics, vol. 17, no. 1, pp. 12-22, 1915.

[22] D. Breaz, S. Owa, and N. Breaz, "A new integral univalent operator," Acta Universitatis Apulensis. Mathematics. Informatics, no. 16, pp. 11-16, 2008.

[23] J. A. Pfaltzgraff, "Univalence of the integral of $f^{\prime}(z)^{\lambda}$," The Bulletin of the London Mathematical Society, vol. 7, no. 3, pp. 254-256, 1975.

[24] N. N. Pascu and V. Pescar, "On the integral operators of KimMerkes and Pfaltzgraff," Mathematica (Cluj), vol. 32 (55), no. 2, pp. 185-192, 1990.

[25] S. Bulut, "A note on the paper of Breaz and Güney," Journal of Mathematical Inequalities, vol. 2, no. 4, pp. 549-553, 2008. 


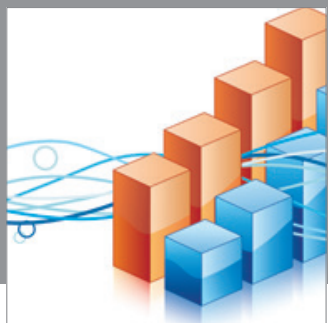

Advances in

Operations Research

mansans

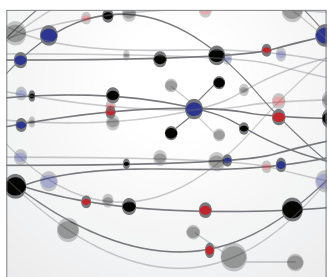

The Scientific World Journal
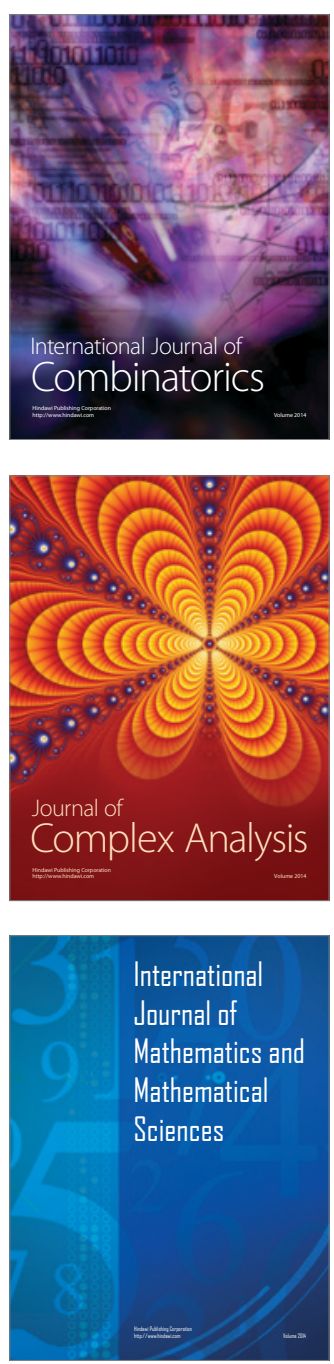
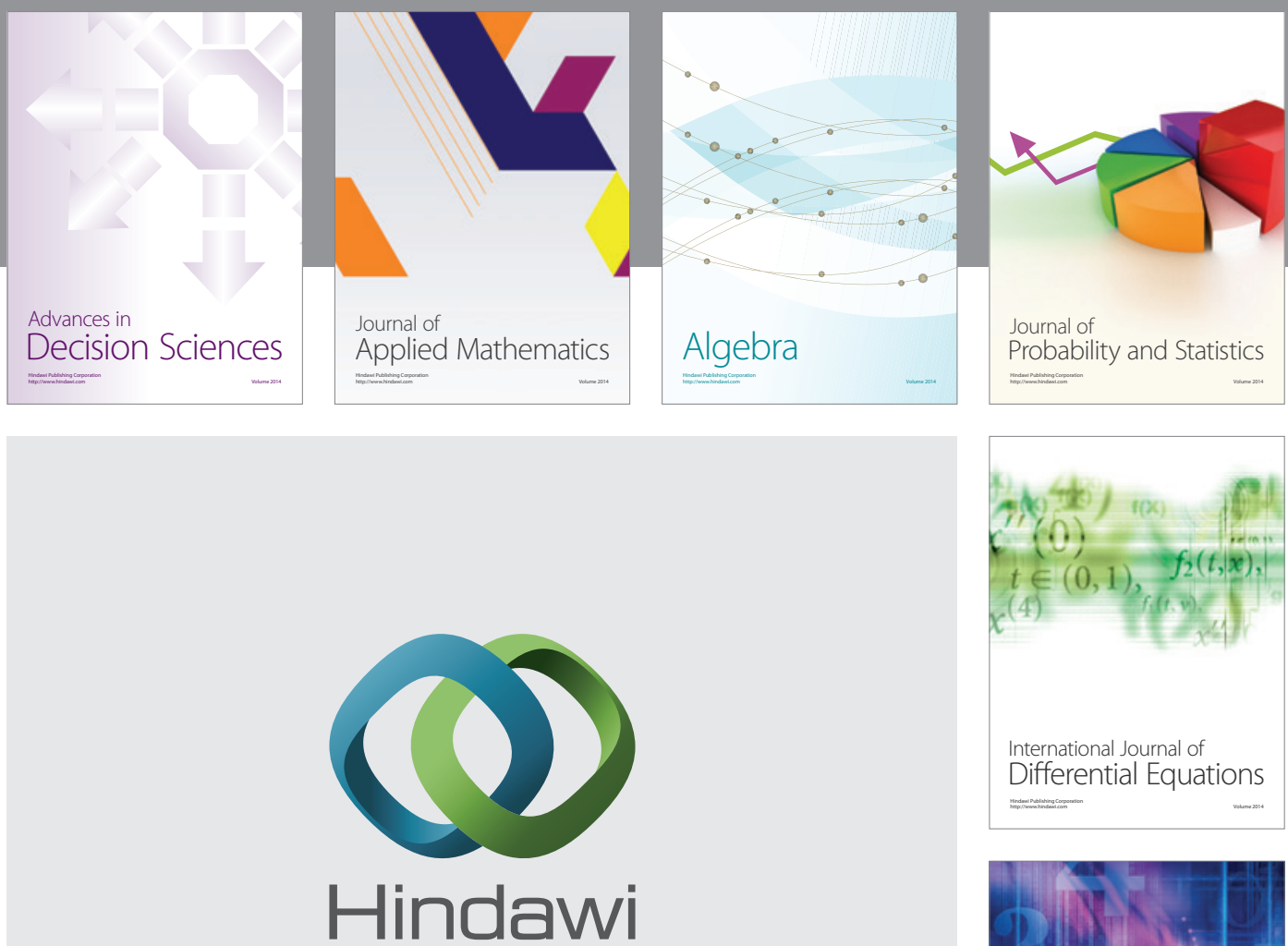

Submit your manuscripts at http://www.hindawi.com
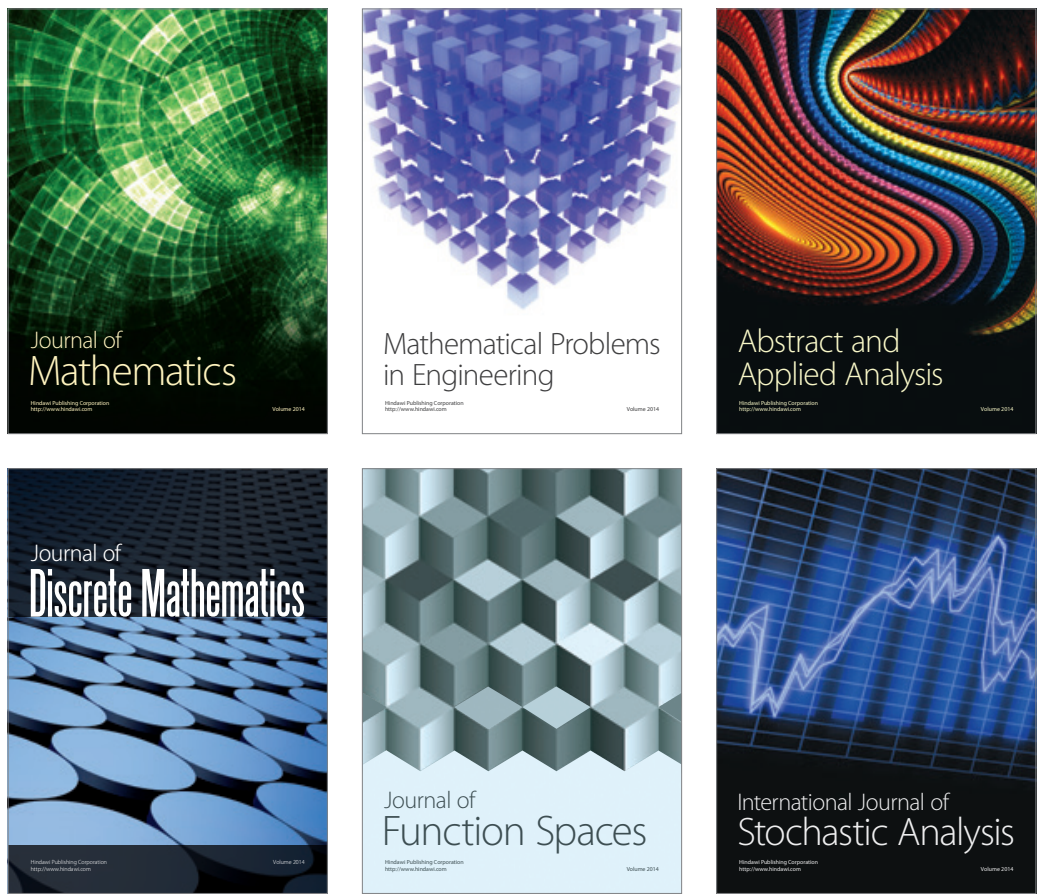

Journal of

Function Spaces

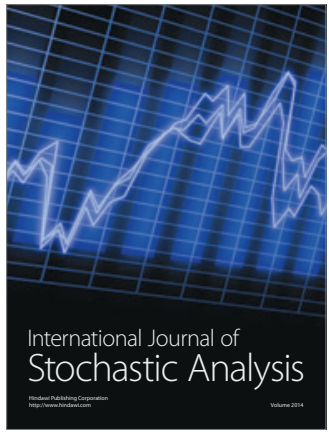

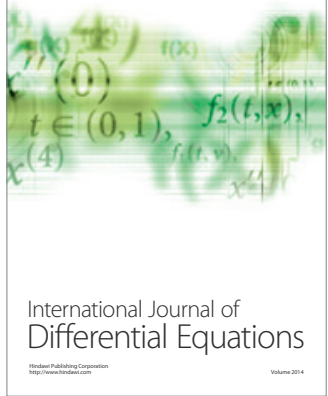
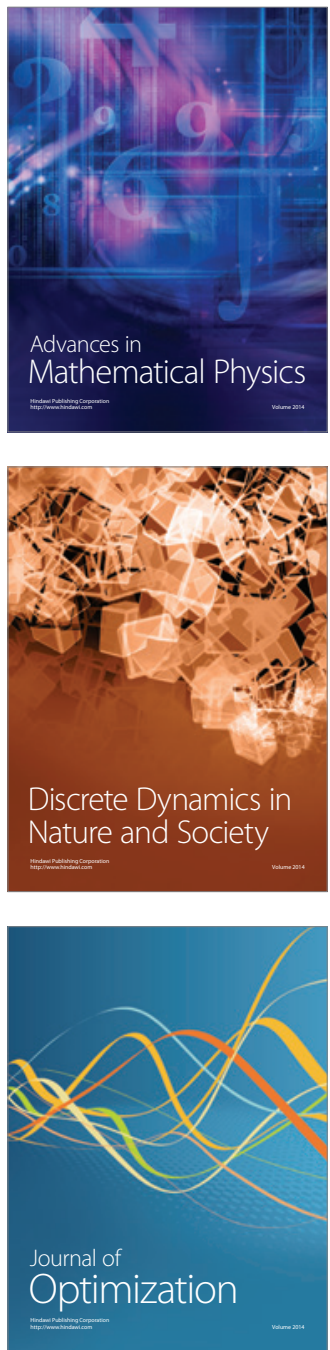